\title{
Extended maceration of must improves phenolic composition and antioxidant potential of Touriga Nacional tropical wine
}

\author{
Erika Samantha Santos de Carvalho ${ }^{\mathrm{a}}$, Aline Telles Biasoto Marques ${ }^{\mathrm{b}^{*}}$, Luiz Claudio Côrrea ${ }^{\mathrm{b}}$, \\ Maria Auxiliadora Coelho de Lima ${ }^{\mathrm{b}}$, Renan Danielskic ${ }^{\mathrm{c}}$ and Janice Izabel Druzian ${ }^{\mathrm{a}}$
}

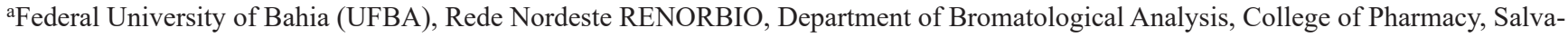
dor, Bahia, CEP 40.171-970, Brazil

bBrazilian Agricultural Research Corporation, Embrapa Tropical Semiárido, Rodovia BR-428, Km 152, Zona Rural - Caixa Postal 23 ,

Petrolina, Pernambuco, CEP 56.302-970, Brazil

'Department of Biochemistry, Memorial University of Newfoundland, St. John's, NL, A1B 3X9, Canada

*Corresponding author: Aline Telles Biasoto Marques, Brazilian Agricultural Research Corporation, Embrapa Tropical Semiárido, Rodovia BR-428, Km 152, Zona Rural - Caixa Postal 23, Petrolina, Pernambuco, CEP 56.302-970, Brazil. E-mail: aline.biasoto@embrapa.br

DOI: $10.31665 /$ JFB.2021.13260

Received: March 26, 2021; Revised received \& accepted: March 30, 2021

Citation: de Carvalho, E.S.S., Marques, A.T.B, Côrrea, L.C., de Lima, M.A.C., Danielski, R., and Druzian, J.I. (2021). Extended maceration of must improves phenolic composition and antioxidant potential of Touriga Nacional tropical wine. J. Food Bioact. 13: 62-73.
\end{abstract}

\begin{abstract}
The "Submédio do Vale do São Francisco" (S.V.S.F.), located in the Brazilian Northeast, is an emerging winemaking region in South America. Touriga Nacional (T.N.) grapes originated in Portugal, thrive under the climatic conditions found in the S.V.S.F. The effects of extended maceration on different parameters of red wine must produced with T.N. grapes from two harvest years from S.V.S.F. were studied. Increased maceration time (16 to 20 days) resulted in greater total phenolic contents ( 40 to $85 \%$ ) compared to eight days of maceration, along with higher antiradical activity (15 to $36 \%$ ). Regardless of the harvest year, the antiradical activity was mostly related to the contents of resveratrol, (+)-catechin, isorhamnetin-3-O-glucosidase, and (-)-epigallocatechin gallate. In contrast, color intensity was not affected. The increase in the maceration period induced a positive effect on the phenolic composition, which was reflected in the higher antiradical activity of T.N. red wine.
\end{abstract}

Keywords: Tropical wines; Touriga Nacional; HPLC-DAD-FD; Extended pre-fermentation maceration; Bioactive composition.

\section{Introduction}

Located in the Brazilian Northeast region, the "Submédio do Vale do São Francisco" (S.S.F.V.) is an emerging winemaking region in South America, focusing on the production of high-quality tropical wines (Pereira et al., 2016; Pereira et al., 2018). Tropical viticulture practices are possible in regions where minimum temperatures are not low enough to induce natural vegetative repose in the vines (Oliveira et., 2019). The S.S.F.V. is located at an altitude of $350 \mathrm{~m}$, between parallels $8^{\circ}$ and $9^{\circ} \mathrm{S}$, with an average temperature of $26^{\circ} \mathrm{C}$, annual precipitation around $500 \mathrm{~mm}$, and $3,000 \mathrm{~h}$ of sunshine per year. The semiarid tropical climate condition, associated with the absence of winter and water availability for irrigation, allows the production of grapes on a large-scale, reaching up to two and a half harvests in the same vineyard per year (Teixeira et al., 2013). Tropical wines began to be vinified in Brazil after advancing table grape production in the S.S.F.V (Camargo et al., 2011).

Touriga Nacional is one of the most prominent Portuguese grape varieties and is currently one of the main cultivars in the S.S.F.V. for red wines, once the vine is fairly vigorous and presents good adaptation to high temperatures and solar irradiance (Jackson, 2003; Oliveira et al., 2019). Touriga Nacional red wines from S.S.F.V. have shown high antioxidant capacity associated with a significant content of phenolic compounds, including abundant concentrations of $(+)$-catechin, (-)-epicatechin, (-)-epicatechin gallate, procyanidin A2, B1 and B2, rutin, gallic acid and stilbenes, as well as the anthocyanins malvidin 3-O-glucoside, peonidin 3-O- 
glucoside, and delphinidin 3-O-glucoside (Padilha et al., 2017a). Besides their well-known antioxidant properties, the mentioned anthocyanins also contribute to the intense color of the Touriga Nacional red wines produced in the S.S.F.V.

Wine composition is directly affected by the conditions in which grapes are processed, including destemming and crushing of the berries, pressing, addition of sulphite, and the maceration step (Lima et al., 2015; Sun et al., 2001; Gomez-Miguez et al., 2007; Ribéreau-Gayon et al., 2006). Maceration, which traditionally occurs along with alcoholic fermentation, is the stage where grape components are extracted. This step depends on the time and temperature in which the skin and seeds are in contact with the wine must. Maceration usually takes less than eight days (Kocabey et al., 2016; Jordão et al., 2001). Therefore, this is one of the crucial steps in winemaking, once phenolic acids and flavonoids found in red wine are generally located in the grape's skins and seeds (Barbará et al., 2019; Rivero et al 2019; Kelebek et al., 2006). High contents of phenolic compounds positively affect the flavor, structure, color, and stability of wine (Granato et al., 2016). Hence, maceration time is a critical factor in obtaining a satisfactory balance among red wine components, also enhancing the product's bioactive potential and antioxidant capacity (Jordão et al., 2001, Jordão et al., 2012; Sun et al., 2001).

Recent findings (Lukic et al., 2016) have compared several wine maceration procedures and looked into the effects of cryomaceration and thermovinification. The study showed discrepant results due to the different grape cultivars that were tested (Lukic et al., 2016). Nevertheless, Yilmaztekin et al. (2015) used extended conventional maceration during fermentation (up to 15 days) to produce wine with grape cultivar Karaoglan from Arapgir (Malatya, Turkey) and obtained a high content of volatile compounds. Alencar et al. (2018) also used extended maceration (up to 30 days) to produce tropical red wine from Syrah grape from the S.S.F.V. region, and the product showed a high concentration of phenolic compounds as well as increased antioxidant capacity.

Although little is known about the effects of extended conventional maceration on red wines produced with Touriga Nacional grape, wine quality is highly influenced by the maceration process. The success of this step depends on the grape cultivar, environmental conditions, and harvest period. Therefore, the objective of this study was to evaluate the influence of extended maceration on the phenolic compounds, antiradical activity, and color intensity of red wines produced from Touriga Nacional grapes grown in tropical conditions and harvested in different seasons.

\section{Material and methods}

\subsection{Feedstock}

Grapes from the cultivar Touriga Nacional (Vitis vinifera L.) from an experimental area $\left(9^{\circ} 2^{\prime} \mathrm{S}, 40^{\circ} 11^{\prime} \mathrm{W}, 365.5 \mathrm{~m}\right.$, Lagoa Grande, Pernambuco, Brazil) composed of 360 plants were harvested (Fig. 1). Each area consisted of 6 plants in each side, totalizing 12 plants. The grapevines grafted under the 'Paulsen 1103' rootstock were conducted in a trellis system with $1.5 \mathrm{~m}$ spacing between plants, being further drip irrigated. The grapes were harvested in two crops in distinct seasons - Vintage 2016 (October 2016-February 2017) and Vintage 2017 (March-July), harvested with 119 and 125 days after pruning, respectively, when the content of soluble solids reached over $24^{\circ}$ Brix. The climatic conditions of the two crops were monitored, as shown in Table S1.

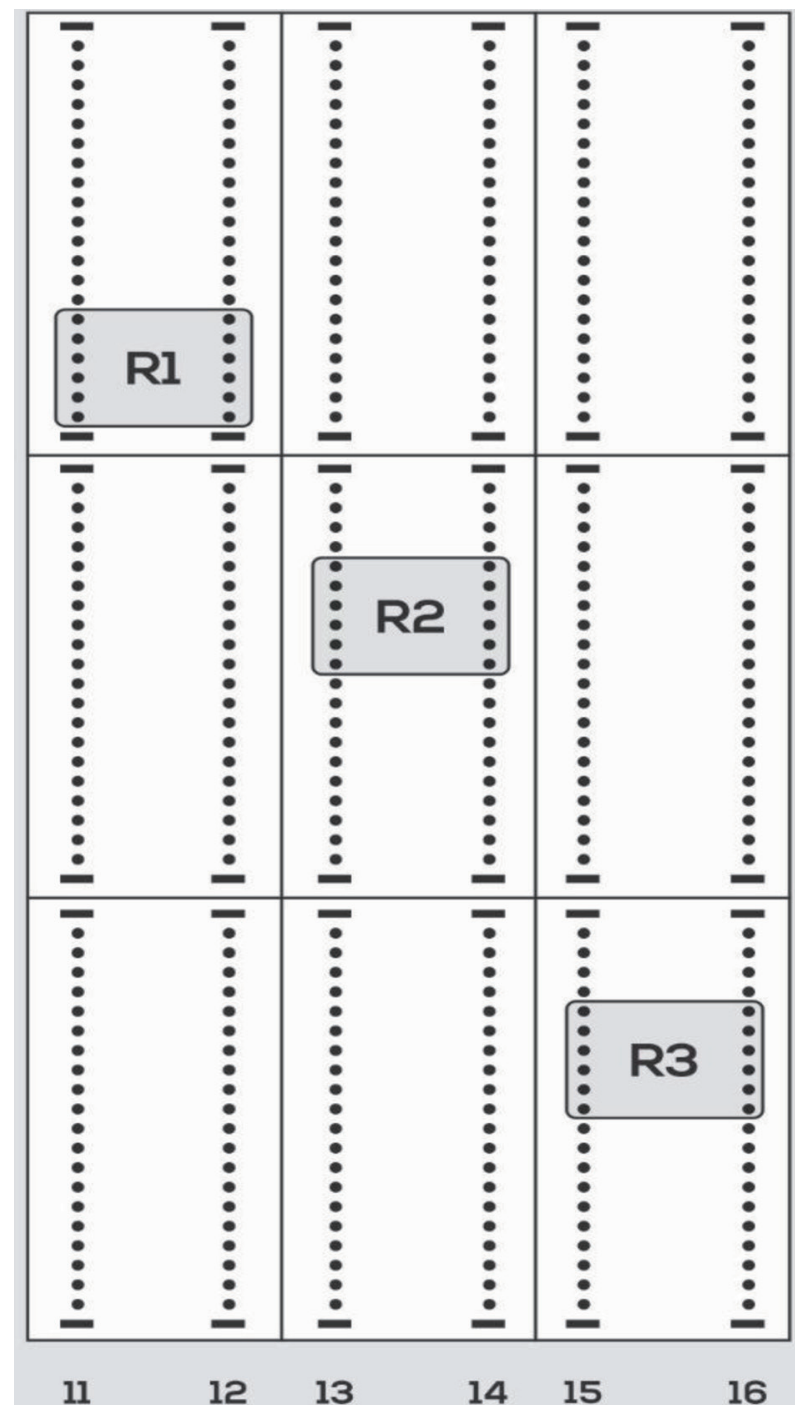

Figure 1. Scheme showing the field experiments that originate the wines used in this study for 2016 and 2017 harvests.

\subsection{Winemaking}

Wines were produced $50 \mathrm{~km}$ away from the harvest site, using traditional winemaking procedures for young red wines (Peynaud, 1997). Twenty-five kilograms of grapes from each experimental area block (R1, R2 and R3) shown in Figure 1 were collected. To obtain the must, grapes from blocks R1, R2, and R3 were destemmed and crushed, promoting a slight pressure on the pomace. Next, each block of must was transferred to three 20 L glass bottles closed with airlock valves. Maceration and fermentation were initiated after the addition of potassium metabisulfite $(0.10$ $\mathrm{g} / \mathrm{L})$, pectinolytic enzyme Everum Thermp Everintec (0.01 g/L), commercial yeast Maurivin PDM Coatec Saccharomyces cerevisiae bavanus $(0.20 \mathrm{~g} / \mathrm{L})$, and ammonium phosphate as a fermentation starter Gesferm plus Coatec $(0.20 \mathrm{~g} / \mathrm{L})$ under controlled temperature $\left(24 \pm 1{ }^{\circ} \mathrm{C}\right)$. During the maceration period, which lasted 20 days, $50 \mathrm{~mL}$ aliquots of must (R1, R2 and R3) were drawn in four days intervals in triplicate (Table 1) and stored in an ultra freezer $\left(-80{ }^{\circ} \mathrm{C}\right)$. 
Table 1. Maceration time planning and samples

\begin{tabular}{lc}
\hline Sample code & Time of maceration (days) \\
\hline P0 & 1 st (must) \\
P1 & 4 \\
P2 & 8 \\
P3 & 12 \\
P4 & 16 \\
P5 & 20 t \\
& Harvest year \\
P0 & 1 st (must) \\
P1 & 4 th \\
P2 & 8 th \\
P3 & 12 th \\
P4 & 16 th \\
P5 & 20 th \\
\hline
\end{tabular}

\subsection{Color intensity (CI) and total phenolic content (TPC)}

Color intensity (CI) was determined spectrophotometrically (Cary 50 Bio U.V./ Visible Spectrophotometer, Palo Alto, CA, USA) using the sum of readings at wavelengths 420,520 , and $620 \mathrm{~nm}$, according to Ribéreau-Gayon et al. (2006). Total phenolic content was determined by the Folin-Ciocalteu method as explained by Singleton and Rossi (1965). Gallic acid was used as a standard to build a calibration curve, and the results were expressed as mg of gallic acid equivalents (GAE)/L of sample.

\subsection{Antioxidant capacity towards DPPH radical and ABTS radi- cal cation}

Antioxidant capacity was determined by the DPPH (Sigma-Aldrich, St. Louis, MO, USA) (1,1-diphenyl-2-picrylhydrazyl) assay according to Brand-Williams et al. (1995). Samples were mixed with an ethanolic solution $(61 \mu \mathrm{M})$ of DPPH and kept in the dark for $30 \mathrm{~min}$. Antioxidant capacity was also evaluated by the scavenging of ABTS (2,2'-azino-bis(3-ethylbenzothiazoline-6-sulfonic acid) radical cation (Sigma-Aldrich, St. Louis, MO, USA), as reported by Mcrae et al. (2019). The absorbances were read at 515 (DPPH assay) and $734 \mathrm{~nm}$ (ABTS assay). Trolox (6-hydroxy2,5,7,8-tetramethylchroman-2-carboxylic acid) was used to build calibration curves for both assays, and the results were expressed as $\mu \mathrm{mol}$ Trolox equivalent (TE)/ $\mathrm{mL}$.

\subsection{Phenolic profile by HPLC-DAD-FD}

Phenolic compounds were identified by high-performance liquid chromatography (Waters Alliance 2695, Milford, MA, USA) with diode array detector (DAD) and fluorescence detector (FD), according to Natividade et al. (2013) and Costa et al (2020). The chromatographic parameters for each phenolic standard used are shown in Table S2. For column separation, a Gemini-NX18 precolumn $\left(4,0 \mathrm{~mm} \times 3,0 \mathrm{~mm}\right.$, Phenomenex ${ }^{\circledR}$, Torrance, CA, USA) and a Gemini-NX18 column (150 mm x 4,60 mm x $3 \mu \mathrm{m}$, Phenomenex ${ }^{\circledR}$, Torrance, CA) were used. Mobile phases were composed of (A) $0.85 \%$ orthophosphoric acid solution and (B) acetonitrile (HPLC grade, J.T. Baker, Phillipsburg, NJ, USA). The flow rate was set to $0.5 \mathrm{~mL} / \mathrm{min}$. The oven (Waters Alliance 2695, Milford, MA, USA) temperature was $40{ }^{\circ} \mathrm{C}$, with an elution gradient composed of: $0 \min 100 \%$ A, $10 \min 93 \%$ A, $20 \min 90 \%$ A, 30 $\min 88 \%$ A, $40 \min 77 \%$ A, $45 \min 65 \%$ A, and $55 \min 100 \%$ B. Sample aliquots were filtered using a nylon membrane of 0.45 $\mu \mathrm{m}\left(\right.$ Phenomenex ${ }^{\circledR}$, Torrance, CA, USA) prior to injection into the system. Samples were injected in triplicate $(10 \mu \mathrm{L})$. Phenolic compounds were detected at 280,320, 360, and $520 \mathrm{~nm}$ using DAD, and with FD (emission of $280 \mathrm{~nm}$ and excitation at $320 \mathrm{~nm}$ ). Data were processed by the software Waters Empower TM 2 (Milford, MA, USA).

For quantification, standard curves were built using phenolic acids, namely gallic, caffeic, $p$-coumaric, and chlorogenic acids (Sigma-Aldrich, St. Louis, MO, USA). Flavonoid standards included kaempferol-3-O-glucoside, isorhamnetin-3-O-glucoside, myricetin, quercetin $3-\beta-\mathrm{D}$-glucoside, rutin, $(+)$-catechin, (-)-epigallocatechin gallate, $(-)$-epicatechin gallate, procyanidin $\mathrm{A} 2$, procyanidin B1, and procyanidin B2. The identification and quantification of anthocyanins were performed according to Costa et al. (2020), using the standards pelargonidin 3-O-glucoside, cyanidin 3-O-glucoside, delphinidin 3-O-glucoside, malvidin 3-O-glucoside, peonidin 3-O-glucoside, and petunidin 3-O-glucoside (Extrasynthese, Genay, France). Stilbenes were also quantified using cis-resveratrol, piceatannol, and trans-resveratrol (Extrasynthese, Genay, France). All the standards presented a purity grade $\geq 98 \%$.

\subsection{Data analysis}

Data were submitted to ANOVA and Tukey means test $(p \leq 0.05)$ by the software SAS ${ }^{\circledR}$ University (S.A.S. Institute Inc, Cary, NC, USA). Predictive models for monitoring the maceration process were built using polynomial regression and the statistical software OriginPro ${ }^{\circledR} 8.0$ (OriginLab Corporation, Northampton, MA, USA). Principal Component Analysis (PCA) was generated using Pearson correlation matrix, additional variables (antioxidant activity values) and the XLStat software (Addinsoft Inc., Anglesey, UK). Additionally, Pearson correlation analysis was also applied to identify the positive and negative correlations between phenolic compounds and antioxidant activity at $5 \%$ level of significance.

\section{Results and discussion}

\subsection{Phenolic profile determined by HPLC-DAD-FD}

The phenolic composition of Touriga Nacional wine is shown in Table 2. A total of 27 phenolic compounds were identified in musts with different maceration times. When comparing the content of each identified phenolic, significant differences $(p \leq 0.05)$ were observed throughout the maceration period and also between the two harvests (within the same maceration time).

The sum of identified phenolic acids varied from 318.58 to $568.65 \mathrm{mg} / \mathrm{L}$ for the 2016 harvest and from 195.71 to $514.34 \mathrm{mg} / \mathrm{L}$ for the 2017 harvest. Gallic acid increased during maceration time up to 16 days in the 2016 harvest, while for the 2017 harvest, both gallic acid and chlorogenic acid presented increasing concentrations up to 20 days of maceration. However, the same was not observed for ferulic, caffeic, and $p$-coumaric acids, which presented 
Table 2. Phenolic compounds $(n=27)$ of Touriga Nacional musts from vintages 2016 and 2017 throughout maceration period

\begin{tabular}{|c|c|c|c|c|c|c|}
\hline \multirow{2}{*}{ Phenolic compounds (mg/L) } & \multicolumn{6}{|c|}{ Maceration time (days) vintage 2016} \\
\hline & PO & P1 & $\mathbf{P 2}$ & P3 & P4 & P5 \\
\hline Gallic acid & $2.50^{d}$ & $11.80^{c d}$ & $24.03^{b c}$ & $34.55^{\mathrm{ab}}$ & $48.58^{\mathrm{a}}$ & $39.28^{a b}$ \\
\hline Caftaric acid & $287.51^{d}$ & $475.87^{a}$ & $488.33^{a}$ & $373.93^{b c}$ & $386.60^{\mathrm{b}}$ & $342.81^{c}$ \\
\hline Chlorogenic acid & $10.05^{b}$ & $10.46^{\mathrm{b}}$ & $13.18^{\mathrm{ab}}$ & $11.28^{\mathrm{b}}$ & $15.35^{\mathrm{a}}$ & $13.78^{\mathrm{ab}}$ \\
\hline Caffeic acid & $16.16^{d}$ & $35.47^{a}$ & $34.53^{a}$ & $25.43^{b}$ & $23.98^{b}$ & $20.50^{c}$ \\
\hline$p$-Coumaric acid & $1.12^{\mathrm{c}}$ & $1.48^{\mathrm{bc}}$ & $1.58^{\mathrm{bc}}$ & $2.12^{\mathrm{b}}$ & $3.49^{a}$ & $3.62^{\mathrm{a}}$ \\
\hline Ferulic acid & $1.23^{c}$ & $6.13^{\mathrm{ab}}$ & $6.98^{\mathrm{a}}$ & $5.36^{\mathrm{ab}}$ & $5.35^{\mathrm{ab}}$ & $4.63^{b}$ \\
\hline Total phenolic acids & $318.58^{d}$ & $541.23^{\mathrm{a}}$ & $568.65^{a}$ & $452.69^{b c}$ & $483.35^{b}$ & $424.64^{c}$ \\
\hline (-)-Epicatechin gallate & $1.88^{b}$ & $2.23^{\mathrm{a}}$ & $1.99^{\mathrm{ab}}$ & $1.86^{\mathrm{ab}}$ & $1.84^{\mathrm{ab}}$ & $1.39^{\mathrm{c}}$ \\
\hline (-)-Epigallocatechin gallate & $2.41^{d}$ & $2.69^{c d}$ & $3.23^{a b c}$ & $3.12^{\mathrm{bc}}$ & $3.74^{\mathrm{ab}}$ & $3.93^{\mathrm{a}}$ \\
\hline$(+)$-Catechin & $1.38^{\mathrm{c}}$ & $5.45^{b}$ & $6.45^{b}$ & $6.41^{b}$ & $7.86^{\mathrm{ab}}$ & $8.25^{\mathrm{a}}$ \\
\hline (-)-Epicatechin & $2.76^{d}$ & $20.63^{c}$ & $48.03^{\mathrm{ab}}$ & $68.25^{b}$ & $72.07^{a}$ & $66.10^{\mathrm{b}}$ \\
\hline Procyanidin A2 & $0.88^{d}$ & $1.23^{\mathrm{c}}$ & $1.45^{\mathrm{bc}}$ & $1.63^{\mathrm{ab}}$ & $1.81^{\mathrm{a}}$ & $1.83^{\mathrm{a}}$ \\
\hline Procyanidin B1 & $6.65^{d}$ & $49.25^{c}$ & $112.50^{\mathrm{bc}}$ & $161.96^{\mathrm{ab}}$ & $168.35^{\mathrm{a}}$ & $155.73^{b}$ \\
\hline Procyanidin B2 & $2.61^{d}$ & $18.63^{c}$ & $40.15^{b}$ & $61.65^{\mathrm{bc}}$ & $67.87^{a}$ & $67.11^{\mathrm{a}}$ \\
\hline Total flavanols & $18.596^{d}$ & $100.12^{c}$ & $213.81^{b}$ & $304.91^{\mathrm{ab}}$ & $323.56^{a}$ & $304.36^{a b}$ \\
\hline cis-Resveratrol & $0.18^{c}$ & $0.48^{\mathrm{b}}$ & $0.66^{a b}$ & $0.65^{a b}$ & $0.74^{\mathrm{a}}$ & $0.66^{\mathrm{ab}}$ \\
\hline Piceatannol & $0.80^{c}$ & $1.20^{\mathrm{bc}}$ & $1.75^{\mathrm{a}}$ & $1.30^{\mathrm{ab}}$ & $1.36^{\mathrm{ab}}$ & $1.23^{\mathrm{bc}}$ \\
\hline trans-Resveratrol & $0.41^{b}$ & $0.83^{\mathrm{ab}}$ & $1.01^{\mathrm{a}}$ & $0.76^{\mathrm{ab}}$ & $0.95^{\mathrm{a}}$ & $0.73^{\mathrm{ab}}$ \\
\hline Total stilbenes & $1.40^{\mathrm{C}}$ & $2.51^{\mathrm{b}}$ & $3.43^{a}$ & $2.71^{\mathrm{ab}}$ & $3.06^{\mathrm{ab}}$ & $2.62^{\mathrm{ab}}$ \\
\hline Kaempferol-3-O-glucoside & $0.51^{b}$ & $0.60^{\mathrm{ab}}$ & $0.63^{\mathrm{a}}$ & $0.61^{\mathrm{ab}}$ & $0.60^{\mathrm{ab}}$ & $0.60^{\mathrm{ab}}$ \\
\hline Quercetin 3- $\beta$-D-glucoside & $3.41^{\mathrm{c}}$ & $6.53^{b c}$ & $8.85^{\mathrm{a}}$ & $7.50^{\mathrm{b}}$ & $8.36^{\mathrm{ab}}$ & $6.31^{b c}$ \\
\hline Isorhamnetin-3-O-glucoside & $1.88^{\mathrm{c}}$ & $6.26^{\mathrm{ab}}$ & $6.86^{\mathrm{a}}$ & $5.48^{\mathrm{bc}}$ & $6.35^{\mathrm{ab}}$ & $5.85^{b}$ \\
\hline Myricetin & $1.00^{c}$ & $2.11^{\mathrm{a}}$ & $1.93^{\mathrm{ab}}$ & $1.66^{\mathrm{ab}}$ & $1.71^{\mathrm{ab}}$ & $1.51^{b c}$ \\
\hline Rutin & $1.33^{c}$ & $6.60^{\mathrm{ab}}$ & $7.33^{\mathrm{a}}$ & $6.50^{\mathrm{ab}}$ & $6.10^{\mathrm{ab}}$ & $5.23^{b}$ \\
\hline Total flavonols & $8.15^{d}$ & $22.11^{b c}$ & $25.61^{a}$ & $21.76^{\mathrm{ab}}$ & $23.13^{b}$ & $19.51^{\mathrm{c}}$ \\
\hline Pelargonidin 3-O-glucoside & $6.81^{\mathrm{e}}$ & $15.16^{\mathrm{ab}}$ & $15.68^{\mathrm{a}}$ & $12.41^{b c}$ & $11.70^{\mathrm{cd}}$ & $8.75^{\text {de }}$ \\
\hline Cyanidin 3-O-glucoside & $1.21^{\mathrm{a}}$ & $0.53^{c}$ & $0.50^{c}$ & $0.93^{b}$ & $0.61^{\mathrm{ab}}$ & $0.61^{\mathrm{ab}}$ \\
\hline Delphinidin 3-O-glucoside & $4.88^{b c}$ & $7.06^{\mathrm{a}}$ & $6.56^{\mathrm{ab}}$ & $4.86^{\mathrm{bc}}$ & $4.11^{c}$ & $3.00^{c}$ \\
\hline Malvidin 3-O-glucoside & $77.11^{d}$ & $227.20^{\mathrm{ab}}$ & $255.40^{\mathrm{a}}$ & $207.11^{\mathrm{b}}$ & $207.30^{\mathrm{b}}$ & $164.55^{c}$ \\
\hline Peonidin 3-O-glucoside & $14.15^{\mathrm{ab}}$ & $15.05^{\mathrm{a}}$ & $14.10^{\mathrm{ab}}$ & $9.68^{\mathrm{abc}}$ & $8.68^{b c}$ & $6.51^{\mathrm{c}}$ \\
\hline Petunidin 3-O-glucoside & $1.42^{\mathrm{d}}$ & $2.40^{\mathrm{bc}}$ & $3.28^{\mathrm{a}}$ & $2.72^{\mathrm{ab}}$ & $2.58^{\mathrm{b}}$ & $1.86^{\mathrm{cd}}$ \\
\hline Total anthocyanins & $105.60^{c}$ & $267.42^{\mathrm{ab}}$ & $295.53^{a}$ & $237.73^{b}$ & $235.00^{b}$ & $185.30^{\mathrm{bc}}$ \\
\hline
\end{tabular}

\begin{tabular}{|c|c|c|c|c|c|c|}
\hline \multirow{2}{*}{ Phenolic compounds (mg/L) } & \multicolumn{6}{|c|}{ Maceration time (days) vintage 2017} \\
\hline & PO & P1 & P2 & P3 & P4 & P5 \\
\hline Gallic acid & $1.01^{d}$ & $9.42^{\mathrm{cd}}$ & $22.74^{b c}$ & $31.54^{b}$ & $48.03^{\mathrm{ab}}$ & $51.88^{\mathrm{a}}$ \\
\hline Caftaric acid & $191.97^{c}$ & $421.91^{\mathrm{ab}}$ & $475.52^{\mathrm{a}}$ & $448.525^{a}$ & $392.66^{\mathrm{ab}}$ & $328.35^{b}$ \\
\hline Chlorogenic acid & $1.01^{\mathrm{c}}$ & $5.28^{\mathrm{bc}}$ & $9.02^{\mathrm{abc}}$ & $9.55^{\mathrm{abc}}$ & $11.24^{\mathrm{ab}}$ & $14.69^{a}$ \\
\hline Caffeic acid & $0.36^{\mathrm{ab}}$ & $0.35^{\mathrm{ab}}$ & $0.39^{a b}$ & $0.16^{b}$ & $0.45^{a}$ & $0.35^{\mathrm{ab}}$ \\
\hline$p$-Coumaric acid & $1.07^{c}$ & $4.26^{\mathrm{ab}}$ & $5.45^{a}$ & $2.67^{b c}$ & $2.47^{b c}$ & $3.10^{\mathrm{bc}}$ \\
\hline
\end{tabular}


Table 2. - (continued)

\begin{tabular}{|c|c|c|c|c|c|c|}
\hline \multirow{2}{*}{ Phenolic compounds (mg/L) } & \multicolumn{6}{|c|}{ Maceration time (days) vintage 2017} \\
\hline & PO & P1 & P2 & P3 & P4 & P5 \\
\hline Ferulic acid & $0.29^{c}$ & $0.70^{\mathrm{b}}$ & $1.20^{\mathrm{a}}$ & $0.76^{b}$ & $0.64^{b}$ & $0.57^{b}$ \\
\hline Total phenolic acids & $195.71^{c}$ & $441.94^{\mathrm{ab}}$ & $514.34^{a}$ & $493.21^{\mathrm{ab}}$ & $455.51^{\mathrm{ab}}$ & $398.97^{b}$ \\
\hline (-)-Epicatechin gallate & $0.37^{c}$ & $0.68^{\mathrm{bc}}$ & $0.94^{\mathrm{b}}$ & $2.51^{\mathrm{a}}$ & $2.10^{\mathrm{ab}}$ & $2.04^{\mathrm{ab}}$ \\
\hline (-)-Epigallocatechin gallate & $1.08^{\mathrm{c}}$ & $2.52^{\mathrm{bc}}$ & $2.91^{\mathrm{ab}}$ & $4.42^{\mathrm{a}}$ & $3.23^{\mathrm{ab}}$ & $3.09^{a b}$ \\
\hline (+)-Catechin & $1.06^{e}$ & $7.75^{d}$ & $23.59^{c d}$ & $32.83^{b c}$ & $48.20^{\mathrm{ab}}$ & $54.56^{a}$ \\
\hline (-)-Epicatechin & $1.29^{\mathrm{e}}$ & $10.80^{\text {de }}$ & $30.48^{c d}$ & $44.26^{b c}$ & $64.20^{\mathrm{ab}}$ & $71.70^{\mathrm{a}}$ \\
\hline Procyanidin A2 & $0.42^{c}$ & $0.90^{\mathrm{b}}$ & $1.45^{\mathrm{ab}}$ & $1.28^{\mathrm{bc}}$ & $1.63^{\mathrm{ab}}$ & $1.73^{a}$ \\
\hline Procyanidin B1 & $1.49^{\mathrm{c}}$ & $9.73^{b}$ & $15.96^{b c}$ & $14.38^{b c}$ & $17.64^{\mathrm{ab}}$ & $19.47^{a}$ \\
\hline Procyanidin B2 & $0.46^{c}$ & $3.55^{c}$ & $13.42^{b c}$ & $23.31^{\mathrm{b}}$ & $45.82^{\mathrm{ab}}$ & $54.94^{\mathrm{a}}$ \\
\hline Total flavanols & $6.20^{\mathrm{e}}$ & $35.95^{\mathrm{de}}$ & $88.77^{c d}$ & $123.02^{b c}$ & $182.84^{\mathrm{ab}}$ & $207.55^{a}$ \\
\hline cis-Resveratrol & $0.25^{b}$ & $0.69^{\mathrm{ab}}$ & $0.82^{\mathrm{a}}$ & $0.69^{\mathrm{ab}}$ & $1.04^{\mathrm{a}}$ & $0.84^{\mathrm{a}}$ \\
\hline Piceatannol & $0.27^{c}$ & $0.42^{b}$ & $0.62^{b}$ & $0.64^{a}$ & $0.65^{a}$ & $0.63^{b}$ \\
\hline trans-Resveratrol & $0.25^{c}$ & $0.59^{\mathrm{ab}}$ & $1.01^{\mathrm{a}}$ & $0.98^{b}$ & $0.58^{\mathrm{ab}}$ & $0.54^{\mathrm{ab}}$ \\
\hline Total stilbenes & $0.78^{c}$ & $1.70^{\mathrm{ab}}$ & $2.45^{\mathrm{a}}$ & $2.32^{\mathrm{ab}}$ & $2.27^{\mathrm{ab}}$ & $2.03^{b}$ \\
\hline Kaempferol-3-O-glucoside & $0.23^{c}$ & $1.02^{b}$ & $1.34^{\mathrm{ab}}$ & $1.84^{\mathrm{a}}$ & $1.45^{\mathrm{ab}}$ & $1.18^{\mathrm{ab}}$ \\
\hline Quercetin 3- $\beta$-D-glucoside & $0.73^{c}$ & $4.26^{\mathrm{b}}$ & $5.71^{\mathrm{a}}$ & $5.39^{\mathrm{ab}}$ & $4.65^{b}$ & $3.56^{\mathrm{bc}}$ \\
\hline Isorhamnetin-3-O-glucoside & $0.65^{c}$ & $6.32^{\mathrm{b}}$ & $8.79^{a}$ & $7.70^{\mathrm{ab}}$ & $7.87^{\mathrm{ab}}$ & $6.57^{\mathrm{b}}$ \\
\hline Myricetin & $0.44^{c}$ & $1.21^{\mathrm{bc}}$ & $2.21^{\mathrm{ab}}$ & $2.78^{a}$ & $2.33^{\mathrm{ab}}$ & $2.03^{\mathrm{ab}}$ \\
\hline Rutin & $0.23^{c}$ & $0.73^{b}$ & $1.08^{\mathrm{ab}}$ & $1.47^{\mathrm{a}}$ & $1.18^{\mathrm{ab}}$ & $1.13^{\mathrm{ab}}$ \\
\hline Total flavonols & $2.29^{c}$ & $13.57^{b}$ & $19.15^{a}$ & $19.19^{a}$ & $17.51^{\mathrm{ab}}$ & $14.48^{\mathrm{ab}}$ \\
\hline Pelargonidin 3-O-glucoside & $5.20^{c}$ & $86.74^{\mathrm{ab}}$ & $115.41^{a}$ & $81.95^{\mathrm{ab}}$ & $70.43^{b}$ & $86.74^{\mathrm{ab}}$ \\
\hline Cyanidin 3-O-glucoside & $2.82^{d}$ & $7.66^{\mathrm{ab}}$ & $8.00^{a}$ & $6.36^{\mathrm{b}}$ & $5.03^{b c}$ & $3.48^{c}$ \\
\hline Delphinidin 3-O-glucoside & $3.50^{c}$ & $66.27^{\mathrm{ab}}$ & $77.45^{\mathrm{a}}$ & $57.37^{\mathrm{ab}}$ & $47.33^{b}$ & $36.45^{b c}$ \\
\hline Malvidin 3-O-glucoside & $27.55^{c}$ & $325.44^{\mathrm{ab}}$ & $267.53^{b}$ & $341.39^{a}$ & $312.99^{a b}$ & $267.02^{b}$ \\
\hline Peonidin 3-O-glucoside & $17.62^{c}$ & $78.07^{\mathrm{ab}}$ & $85.17^{\mathrm{a}}$ & $64.78^{\mathrm{ab}}$ & $50.76^{\mathrm{abc}}$ & $40.04^{b c}$ \\
\hline Petunidin 3-O-glucoside & $0.67^{d}$ & $17.21^{\mathrm{ab}}$ & $20.33^{a}$ & $15.29^{\mathrm{abc}}$ & $11.90^{\mathrm{bc}}$ & $9.33^{c}$ \\
\hline Total anthocyanins & $57.38^{d}$ & $581.14^{\mathrm{a}}$ & $573.91^{\mathrm{ab}}$ & $567.18^{b}$ & $498.47^{b c}$ & $417.62^{c}$ \\
\hline
\end{tabular}

${ }^{1}$ Means with the same lowercase letter in the same lines do not differ significantly according to Tukey's test $(p \leq 0.05) . P 0=1 \mathrm{st}(\mathrm{must}), \mathrm{P} 1=4$ th, $\mathrm{P} 2=8$ th, $\mathrm{P} 3=12$ th, $\mathrm{P} 4=16$ th, $\mathrm{P} 5=20$ th.

an increase up to day 8 of maceration in both harvests. From this point on, the content of these phenolic acids was reduced until day 20 (Table 3). The behavior of caffeic and chlorogenic acids during maceration in the 2016 harvest can be due to the reaction between caffeic, quinic, shikimic, and tartaric acids to form phenolic acid esters, such as chlorogenic acid (Kosseva et al., 2017). The concentrations of gallic, ferulic, and $p$-coumaric acids found in the present study were similar to those reported by Alencar et al. (2018).

Besides its antioxidant activity, chlorogenic acid is also potentially associated with health-promoting effects. These benefits include inhibition of liver cancer cells and potential prevention of type 2 diabetes and cardiovascular ailments (Garambone et al., 2008).

During the maceration procedure, gallic acid is extracted from grape seeds after hydrolysis of gallate esters from flavonoids by the action of esterases. Subsequently, it is esterified with ethanol, forming ethyl gallate. This chemical process seems to also take place during fermentation, benefiting wine ageing (Lingua et al., 2016). According to Kocabey et al. (2016), maceration of grape cv. Karaoglan (Malatya, Turkey) for up to 15 days released $7.15 \mathrm{mg} / \mathrm{L}$ of $p$-coumaric acid and $1.62 \mathrm{mg} / \mathrm{L}$ of ferulic acid. The interaction between phenolic compounds may strongly influence wine quality, even though they are present in minor amounts (Moreno-Arribas and Polo, 2008).

The total flavanol content, from the start to day 20, varied from 100.12 to $304.91 \mathrm{mg} / \mathrm{L}$ in must samples for the 2016 harvest and from 6.20 to $207.55 \mathrm{mg} / \mathrm{L}$ in must for the 2017 harvest (Table 2). Regarding the flavanols identified in the 2016 sample, (-)-epicatechin gallate, (-)-epicatechin, procyanidin B1, and procyanidin B2 showed increasing levels up to 16 days of maceration, while the concentrations of $(-)$-epigallocatechin gallate, $(+)$-catechin, and 
Table 3. Pearson correlation analysis $(p \leq 0.05)$ results for phenolic compounds and antioxidant activity of Touriga Nacional musts from 2016 and 2017 harvests

\begin{tabular}{|c|c|c|c|c|c|c|c|c|}
\hline \multirow{2}{*}{ Phenolic Compounds } & \multicolumn{2}{|c|}{ DPPH (Vintage 2016) } & \multicolumn{2}{|c|}{ ABTS (Vintage 2016) } & \multicolumn{2}{|c|}{ DPPH (Vintage 2017) } & \multicolumn{2}{|c|}{ ABTS (Vintage 2017) } \\
\hline & Pearson $r$ & p-Value & Pearson $r$ & p-Value & Pearson $r$ & $p$-Value & Pearson $r$ & p-Value \\
\hline \multicolumn{9}{|l|}{ Phenolic acids } \\
\hline Gallic acid & 0.93 & $0.01 *$ & 0.87 & $0.03 *$ & 0.84 & $0.04 *$ & 0.83 & $0.04 *$ \\
\hline Caftaric acid & 0.70 & 0.12 & 0.76 & 0.08 & 0.24 & 0.65 & 0.38 & 0.46 \\
\hline Chlorogenic acid & 0.76 & 0.08 & 0.80 & 0.06 & -0.22 & 0.67 & 0.02 & 0.98 \\
\hline Caffeic acid & 0.24 & 0.64 & 0.39 & 0.45 & 0.89 & $0.02 *$ & 0.88 & $0.02 *$ \\
\hline$p$-Coumaric acid & 0.72 & 0.11 & 0.71 & 0.11 & 0.35 & 0.50 & 0.47 & 0.35 \\
\hline Ferulic acid & 0.67 & 0.15 & 0.77 & 0.07 & 0.49 & 0.32 & 0.62 & 0.19 \\
\hline \multicolumn{9}{|l|}{ Flavanols } \\
\hline (-)-Epicatechin gallate & -0.49 & 0.32 & -0.47 & 0.35 & 0.88 & $0.02 *$ & 0.77 & 0.07 \\
\hline (-)-Epigallocatechin gallate & 0.79 & 0.06 & 0.81 & $0.05^{*}$ & 0.94 & $0.01 *$ & 0.84 & $0.03 *$ \\
\hline$(+)$-Catechin & 0.94 & $0.00 *$ & 0.98 & $0.00 *$ & 0.83 & $0.04 *$ & 0.82 & $0.04 *$ \\
\hline (-)-Epicatechin & 0.96 & $0.00 *$ & 0.88 & $0.02 *$ & 0.84 & $0.04^{*}$ & 0.82 & $0.04 *$ \\
\hline Procyanidin A2 & 0.95 & $0.00 *$ & 0.91 & $0.01^{*}$ & 0.89 & $0.02 *$ & 0.94 & $0.00 *$ \\
\hline Procyanidin B1 & 0.95 & $0.00 *$ & 0.87 & $0.02 *$ & 0.92 & $0.01 *$ & 0.96 & $0.00 *$ \\
\hline Procyanidin B2 & 0.93 & $0.01^{*}$ & 0.86 & $0.03 *$ & 0.73 & 0.10 & 0.71 & 0.11 \\
\hline \multicolumn{9}{|l|}{ Stilbenes } \\
\hline cis-Resveratrol & 0.98 & $0.00 *$ & 0.94 & $0.00^{*}$ & 0.86 & $0.03 *$ & 0.95 & $0.00 *$ \\
\hline Piceatannol & 0.61 & 0.20 & 0.67 & 0.14 & 0.96 & $0.00 *$ & 0.98 & $0.00 *$ \\
\hline trans-Resveratrol & 0.70 & 0.12 & 0.79 & 0.06 & 0.68 & 0.14 & 0.68 & 0.13 \\
\hline \multicolumn{9}{|l|}{ Flavonols } \\
\hline Kaempferol-3-O-glucoside & 0.97 & $0.00 *$ & 0.95 & $0.00^{*}$ & 0.95 & $0.00 *$ & 0.90 & $0.01 *$ \\
\hline Quercetin 3- $\beta$-D-glucoside & 0.69 & 0.13 & 0.76 & 0.08 & 0.82 & $0.05^{*}$ & 0.86 & $0.03^{*}$ \\
\hline Isorhamnetin-3-O-glucoside & 0.78 & 0.07 & 0.89 & $0.02 *$ & 0.88 & $0.02 *$ & 0.94 & $0.01 *$ \\
\hline Myricetin & 0.53 & 0.28 & 0.64 & 0.17 & 0.95 & $0.00 *$ & 0.93 & $0.01 *$ \\
\hline Rutin & 0.80 & 0.05 & 0.85 & $0.03 *$ & 0.97 & $0.00 *$ & 0.92 & $0.01 *$ \\
\hline \multicolumn{9}{|l|}{ Anthocyanins } \\
\hline Pelargonidin 3-O-glucoside & 0.50 & 0.32 & 0.59 & 0.21 & 0.66 & 0.16 & 0.74 & 0.09 \\
\hline Cyanidin 3-O-glucoside & -0.84 & $0.03 *$ & -0.92 & $0.01 *$ & 0.33 & 0.52 & 0.40 & 0.43 \\
\hline Delphinidin 3-O-glucoside & -0.19 & 0.71 & -0.10 & 0.84 & 0.60 & 0.21 & 0.66 & 0.15 \\
\hline Malvidin 3-O-glucoside & 0.67 & 0.15 & 0.76 & 0.08 & 0.87 & $0.02 *$ & 0.83 & $0.04 *$ \\
\hline Peonidin 3-O-glucoside & -0.64 & 0.17 & -0.55 & 0.26 & 0.47 & 0.35 & 0.53 & 0.28 \\
\hline Petunidin 3-O-glucoside & 0.81 & 0.05 & 0.79 & 0.06 & 0.59 & 0.21 & 0.66 & 0.16 \\
\hline DPPH & 1.00 & $0.00 *$ & 0.96 & $0.00 *$ & 1.00 & $0.00 *$ & 0.96 & $0.00 *$ \\
\hline ABTS & 0.96 & $0.00 *$ & 1.00 & $0.00 *$ & 0.96 & $0.00 *$ & 1.00 & $0.00 *$ \\
\hline
\end{tabular}

*Valor $\mathrm{p} \leq 0,05$

procyanidin A2 continued to increase during the entire maceration period (20 days). However, for the 2017 samples, the levels of all flavanols consistently increased throughout the 20 days of maceration. Among flavanols, procyanidin $\mathrm{B} 1,(-)$-epicatechin, procyanidin B2, and $(+)$-catechin presented the highest concentrations between 16 and 20 days of maceration for both harvests (Table 2).
According to Lingua et al. (2016), (+)-catechin and (-)-epicatechin are mainly concentrated in grape skins and seeds. In the present study, both compounds reached their concentration peaks around 16-20 days of maceration. Alencar et al. (2018) reported that after 20 days of maceration, cv. Syrah must contained 14.5 $\mathrm{mg} / \mathrm{L}$ of (-)-epicatechin and $5.6 \mathrm{mg} / \mathrm{L}$ of $(+)$-catechin. These re- 
sults are significantly lower than those obtained in the present study, indicating the high bioactive potential of wines produced with Touriga Nacional grapes grown in the S.V.S.F. region.

Procyanidin B1 has been reported as the main flavanol present in grape skin, while the seeds are abundant in procyanidin B2 (González-Manzano et al., 2004), in agreement with the results obtained in the present study (Table 2). Procyanidins are related to flavor, astringency, bitterness, oxidation, and color stability in wines, as well as a myriad of health benefits due to their potential as antioxidant and anti-inflammatory substances (Garrido and Borges, 2013; Kennedy, 2008; Vauzour et al., 2010).

The stilbenes cis-, trans-resveratrol, and piceatannol were identified in musts from both harvests (Table 2). Interestingly, after eight days of maceration, the levels of total stilbenes did not undergo drastic changes as opposed to what was observed for phenolic acids and flavanols (Table 2).

Maceration for 16 days resulted in the highest concentrations of cis-resveratrol for the $2016(0.74 \mathrm{mg} / \mathrm{L})$ and $2017(1.04 \mathrm{mg} / \mathrm{L})$ samples. However, for trans-resveratrol, the highest yield was achieved after eight maceration days in both harvests $(1.01 \mathrm{mg} / \mathrm{L})$. Padilha et al. (2017b) reported a higher trans-resveratrol content for Touriga Nacional wine from S.V.S.F. (3.0 mg/L). Granato et al. (2016) reported trans-resveratrol levels ranging from 1.02 to 4.30 $\mathrm{mg} / \mathrm{L}$ in wines from temperate climate regions, namely the South of Brazil, Argentina, and Chile. On the other hand, cis-resveratrol results were in agreement with wines produced with the cultivars Syrah, Tannat, Cabernet Sauvignon, and Merlot from the S.V.S.F. region (Lucena et al., 2010). The discrepancy in results from several studies can be explained by differences in winemaking practices and climate conditions (Lima et al.,2015). The presence of resveratrol in Touriga Nacional wine is beneficial as this compound is related to cardioprotective and anticarcinogenic effects due to its antioxidant properties (Fernandez-Mar et al., 2012; Vauzour et al., 2010).

Must samples did not benefit from extended maceration regarding total flavonol content, as opposed to phenolic acids and flavanols (Table 2). Baseline concentrations of total flavonols were $8.15 \mathrm{mg} / \mathrm{L}$ for the 2016 harvest and $2.29 \mathrm{mg} / \mathrm{L}$ for the 2017 harvest, reaching $25.61 \mathrm{mg} / \mathrm{L}$ (2016) and $19.15 \mathrm{mg} / \mathrm{L}$ (2017) after eight maceration days, with no significant variations until 20 days of maceration, where the total flavonol content decreased to 19.51 $\mathrm{mg} / \mathrm{L}$ (2016) and $14.48 \mathrm{mg} / \mathrm{L}$ (2017). Quercetin-3-O-glucoside and isorhamnetin-3-O-glucoside were identified in significant amounts, while kaempferol-3-O-glucoside, rutin, and myricetin were present in lower concentrations. According to Casassa et al. (2013), flavonols are progressively extracted during the first five to nine days of maceration. From this point on, these molecules undergo acid hydrolysis, releasing unstable aglycones that may be lost by ethanolic precipitation. Flavonols from grape skin are yellow compounds released during the extraction process. In wines, they are usually responsible for flavor features, such as astringency and bitterness (Tsanova-Savova and Ribarova, 2012; Monagas et al., 2003). Although extended maceration results in higher contents of phenolic acids and flavanols, flavonols do not change in the same way, which prevents the wine from altering its sensory characteristics. Conjugated flavonols have a more significant presence than their free counterparts, as reported by Tsanova-savova and Ribarova (2012) for Bulgarian Merlot and Cabernet wines.

Total anthocyanins were significantly reduced with extended maceration (8-20 days), ranging from 295.53 to $185.30 \mathrm{mg} / \mathrm{L}$ for the 2016 samples, and from 573.91 to $417.62 \mathrm{mg} / \mathrm{L}$ for the 2017 samples (Table 2). Overall, six anthocyanins were identified in the must (pelargonidin 3-O-glucoside, cyanidin 3-O-glucoside, delphinidin 3-O-glucoside, malvidin 3-O-glucoside, peonidin 3-O- glucoside, and petunidin 3-O-glucoside), increasing their amounts up to day 8 of maceration (Table 2).

The decline in anthocyanin content after eight days of maceration has been attributed to ionic adsorption by negatively charged yeasts, adsorption in bitartrate crystals, and incorporation in polymeric pigments (Harbertson and Spayd, 2006). Anthocyanin reduction might cause decreased co-pigmentation in wines as a result of the increase in the must's ethanol concentration due to fermentation. This causes the medium to become more hydrophobic, contributing to anthocyanin loss and, consequently, conferring a less appealing color (Hermosin-Gutiérrez, 2003). Therefore, it is noteworthy that the present study showed a lower malvidin-3-Oglucosidase concentration than Touriga Nacional wine (888.31 $\mathrm{mg} / \mathrm{L}$ ) produced after nine maceration days in the Viseu region, located in the North of Portugal (Jordão et al., 2012).

\subsection{Predictive models}

The effect of maceration (up to 20 days) on color intensity, total phenolic content, antioxidant capacity (DPPH and ABTS), and specific phenolic classes (total phenolic acids, total flavanols, total stilbenes, total anthocyanins, and total flavonols) of Touriga Nacional wine are summarized in Figure 2. For each variable, the predictive models obtained high coefficients of determination $\left(\mathrm{R}^{2}\right.$ ), varying from 0.844 (color intensity) to 0.997 (total anthocyanins) for the 2016 harvest and from 0.643 (total flavonols) to 0.986 (color intensity) for the 2017 harvest.

The model in Figure 2a $\left(\mathrm{R}^{2} 0.844, \mathrm{p}=0.005\right)$ for the 2016 harvest showed a higher color intensity between four and 12 days of maceration, with no large variations observed until the end of the maceration period. Samples from the 2017 harvest $\left(R^{2}\right.$ 0.986, p $\geq 0.004$ ) followed the same trend. Gil et al. (2012) studied extended maceration (up to 30 days) for Tempranillo and Cabernet Sauvignon wines and found a similar trend to the one detected in the present study, with the color intensity stabilizing after ten days of maceration. However, the Touriga Nacional cultivar has shown greater potential for color extraction ( 24.0) when compared to the cultivars Tempranillo and Cabernet Sauvignon (14.1 and 13.5, respectively). Gomez-Miguez et al. (2007) and McRae et al. (2019) observed that color intensity and total phenolic content increased after four days of maceration. In the present study, after eight days of maceration, color intensity remained relatively constant. This is usually the maceration period used in standard commercial wines.

As shown in Figure 2b, an increase of $45 \%$ in total phenolic content was observed from day 8 to 16 of maceration in the 2016 must. After 16 days, the concentration declined. On the other hand, the must from the 2017 harvest $\left(R^{2} 0.948, p=0.012\right)$ registered an increase in phenolic concentration from 16 to 20 days of maceration, which evidences the influence of harvest on this bioactive group. Alencar et al. (2018) studied extended maceration (up to 30 days) to produce Syrah wine and observed that total phenolic content was higher after 15 days of maceration. These results show that phenolics are more affected by extended maceration than color intensity in tropical wines. Phenolic content and antioxidant activity are influenced by environmental factors, such as grape cultivar, climatic conditions, area of cultivation, and winemaking conditions (Gómez-Cordovés, Faitová et al., 2004; De Coninck et al., 2006; Gonçalves and Jordão, 2009).

The antioxidant capacity of Touriga Nacional must, determined by DPPH and ABTS assays, presented significant variations throughout the maceration period for both harvests, as shown in Figure $2 c\left(R^{2} 0.810, p=0.083\right.$ for the 2016 harvest; $R^{2} 0.972, p$ $=0.033$ for the 2017 harvest $)$ and Figure $2 \mathrm{~d}\left(\mathrm{R}^{2} 0.978, \mathrm{p}=0.001\right.$ 

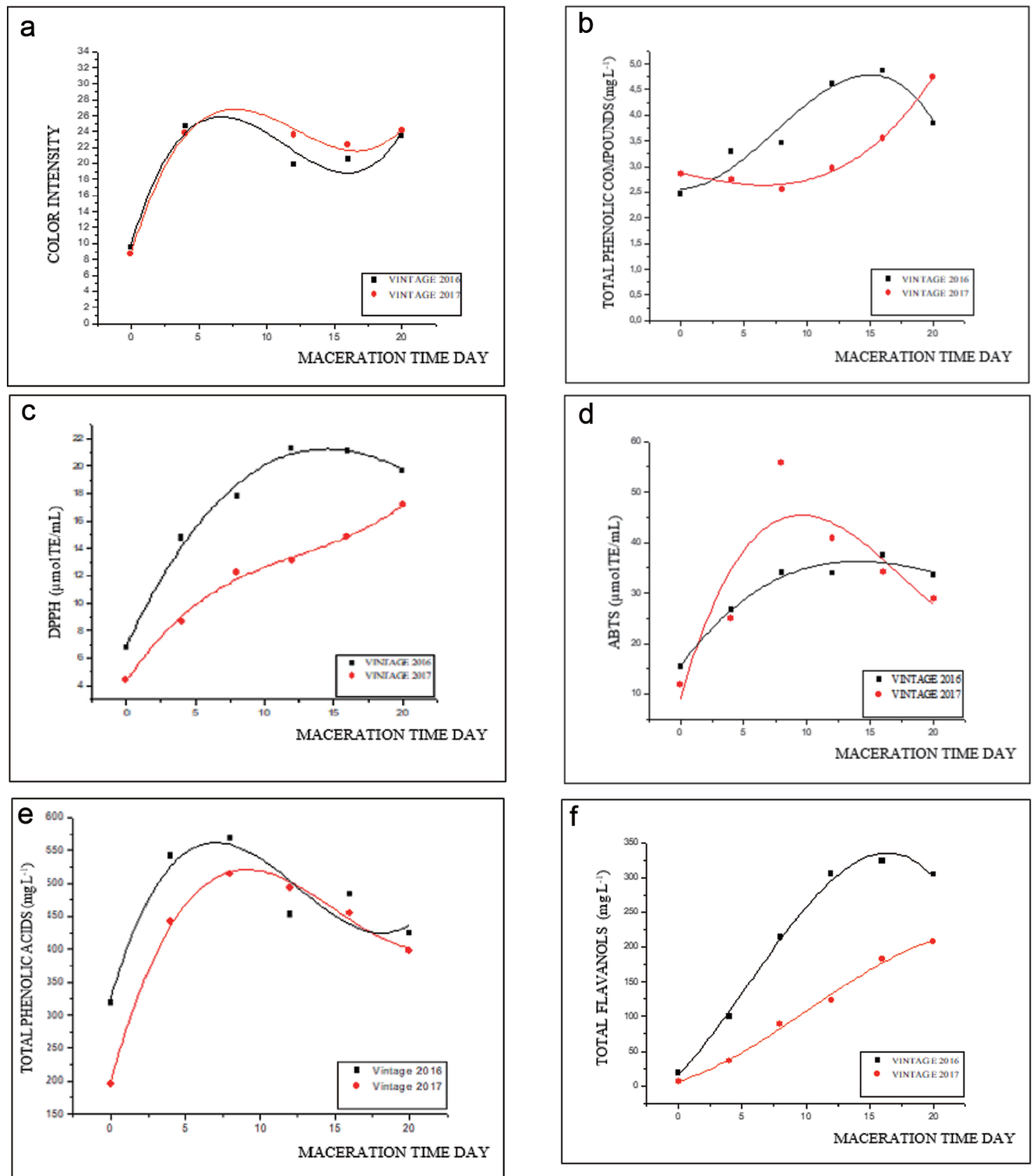

Figure 2. Predictive models for the maceration time of Touriga Nacional red must regarding color intensity (a), Total phenolic content (b), Antioxidant capacity expressed by the radical DPPH (c) and ABTS (d), Total phenolic acids (e), Total flavanols (f), Total Stilbenes (g), Total anthocyanins (h) and Total Flavonols (i). *Equations for the predictive models can be found in Table S3. 
Figure 2. - (continued)
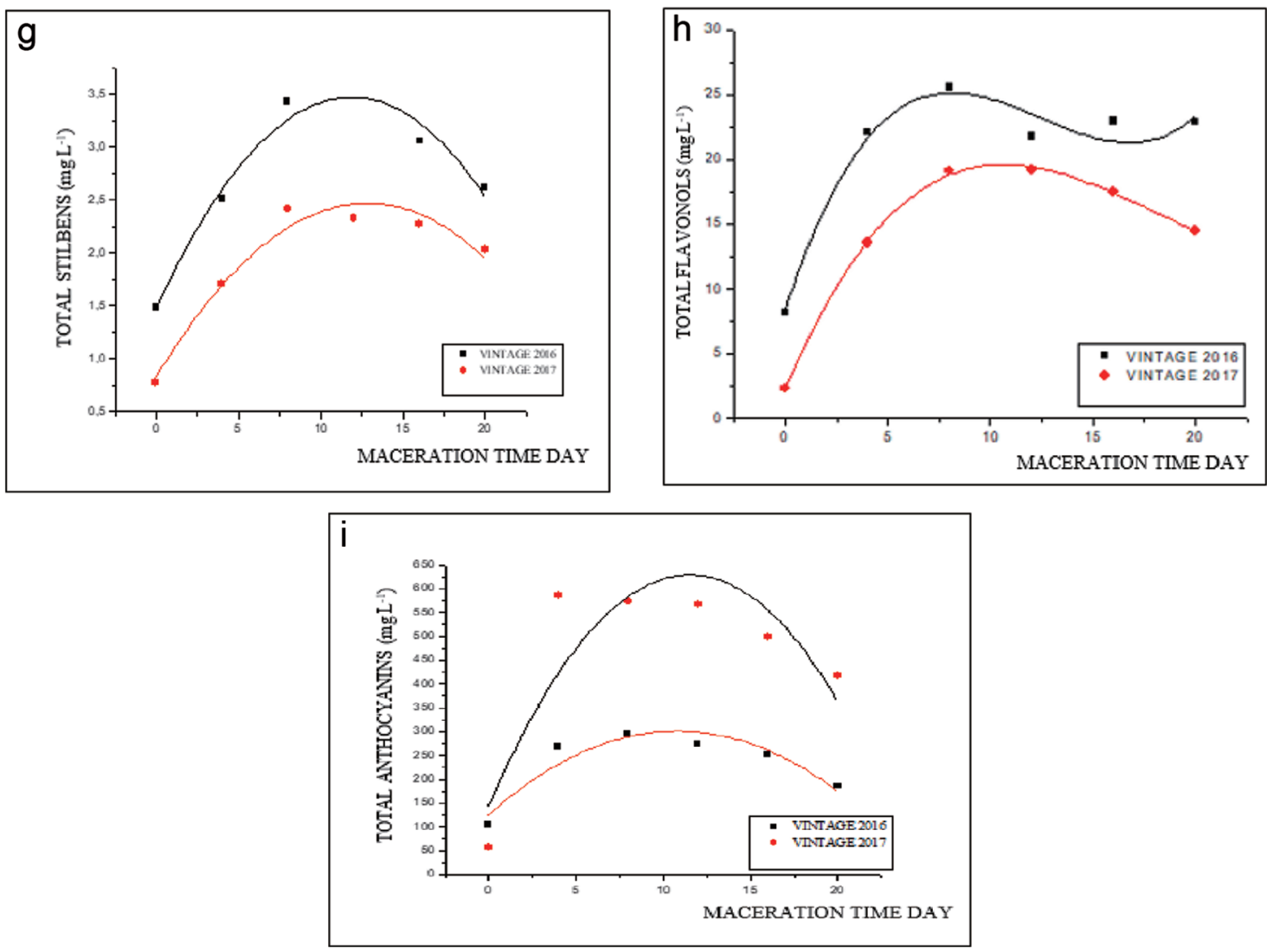

for the 2016 harvest; $\mathrm{R}^{2} 0.983, \mathrm{p}=0,007$ for the 2017 harvest). Regarding the sample from the 2016 harvest, DPPH radical scavenging capacity peaked at 12 days of maceration, while the highest efficiency for ABTS radical scavenging capacity was observed at 8 days of maceration, then declining until the end of the maceration period. Contrarily, must from the 2017 harvest displayed an increasing trend for DPPH radical scavenging activity throughout the entire maceration period. Regarding ABTS radical cation scavenging activity, the peak was reached between 10 and 15 days of maceration, with no further variations for the rest of the maceration period.

Alencar et al. (2018) observed that the DPPH radical scavenging activity of wine produced from Syrah grapes grown in the Brazilian Northeast was $16.00 \mu \mathrm{mol}$ TE/L by using 15 days of maceration. This result is similar to that found in the present study. According to Leeuw et al. (2014), the antioxidant activity determined by the DPPH assay for wines produced from Cabernet Sauvignon, Merlot, Syrah, Pinot Noir and Malbec produced in France, Italy, United Kingdom, United States, Chile, Australia, and Argentina varied from 3.71 to $7.67 \mu \mathrm{mol} \mathrm{TE} / \mathrm{L}$.

Predictive models for specific phenolic classes have also been obtained in the present study. The model for phenolic acids shown in Figure 2e $\left(R^{2} 0.925, p=0.012\right.$ for the 2016 harvest; $R^{2} 0.822$, $p \geq 0.042$ for the 2017 harvest) demonstrates that, regardless of the harvest, phenolic acids are most efficiently extracted by eight days of maceration, decreasing the concentration after this period. Meanwhile, total flavanols reached their highest amount at 16 days of maceration in samples from the 2016 harvest, while total flavanol levels continued to increase up to 20 days of maceration in must from the 2017 harvest (Fig. 2f). Stilbenes (Fig. 2g), anthocyanins (Fig. 2i) and flavonols (Fig. 2h) followed a similar trend by decreased levels after reaching the peak. All these phenolic classes were more predominant in the must from the 2016 harvest compared to those from the 2017 harvest. The presence of several phenolic classes and the interaction between different compounds play an essential role in the antioxidant capacity of wine, related to the protection against oxidative stress in the human body (Oroian and Escriche, 2015; Roleira et al., 2015, Gris et al., 2013; Samoticha et al., 2017).

\subsection{Analysis of Pearson correlation}

Figure 3 presents the principal component analysis representing the interaction between the phenolic profile and antiradical activity of must samples from the 2016 and 2017 harvests.

The extended mapping explains approximately $77.36 \%$ of the variation among phenolic compounds and antiradical activity, mainly due to phenolic compounds' presence on the right of PC1 $(40.88 \%)$. Among these phenolics, there are phenolic acids, stilbenes, flavonols, flavanols, and anthocyanins. Sample distribution (Fig. 3b) shows the formation of four different groups, as follows - group 1 (SF17P1), group 2 (SF16P0 and SF17P0), group 3 (2016 harvest from P1 to P5), and group 4 (2017 harvest from P2 to P5). Group 3 samples showed a high correlation with phenolics located on the left side of PC1, indicating the group's rich concentration in such compounds. The same can be said for group 4 samples, highly correlated with compounds located on the right side of PC1. 

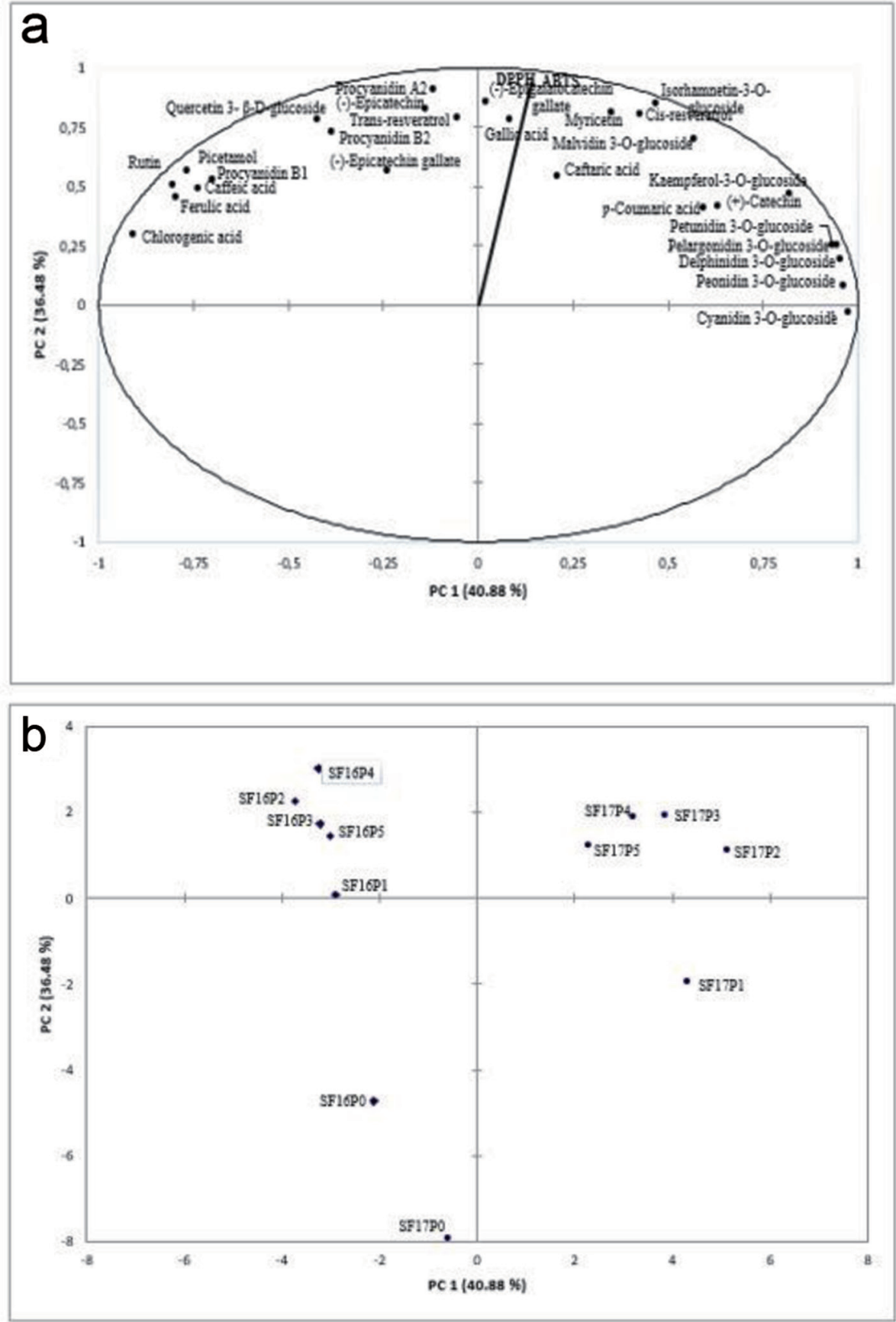

Figure 3. PCA representing (a) DPPH ( $\mu \mathrm{mol} T E / L)$ and ABTS ( $\mu \mathrm{mol} T E / L)$ and phenolic compounds $(n=27)$; (b) the special representation of the samples.

On the other hand, group 2 samples did not show correlation with any compound, which may indicate their lower concentration of phenolics compared to the samples that have undergone maceration. This once again shows the importance of the maceration step for the presence of phenolic compounds in wines.

Maceration periods within group 3 were associated primarily with higher contents of chlorogenic acid, ferulic acid, caffeic acid, flavonols (rutin and quercetin-3- $\beta$-D-glucoside), flavanols (procyanidin A2, B1, B2, (-)-epicatechin, (-)-epigallocatechin gallate), and stilbenes (piceatannol, trans-resveratrol). On PC 2 (36.48\%), the samples SFP2, SFP3, SFP4, and SFP5 from the 2017 harvest demonstrated a greater interaction between antiradical activity and anthocyanins (pelargonidin 3-O-glucoside, delphinidin 3-Oglucoside, malvidin 3-O-glucoside, peonidin 3-O-glucoside, and petunidin 3-O-glucoside), phenolic acids (gallic, caftaric, and $p$ coumaric), and flavonoids (myricetin, kaempferol-3-O-glucoside, isorhamnetin-3-O-glucoside, $(-)$-epicatechin gallate, $(+)$-catechin, and cis-resveratrol) contents. These results confirm the effect of harvest on the content of phenolic compounds.

Table 3 shows the correlation between antioxidant capacity 
(DPPH and ABTS) and phenolic profile in must from Touriga Nacional grapes. Among all phenolic acids, gallic acid showed the highest correlation with antioxidant activity for samples from both harvests. For the 2017 harvest, caffeic acid presented a significant correlation with antioxidant activity $(\mathrm{r}=0.89$ for DPPH and $\mathrm{r}=$ 0.88 for ABTS).

In general, flavanols showed a high positive correlation with antiradical activity in samples from both harvests, with the only exception being (-)-epicatechin gallate in the 2016 must (Table 3 ). Among stilbenes, cis-resveratrol had the highest correlation with antiradical activity in both harvests. A high correlation pattern was also observed for flavonols, except for myricetin in the 2016 must.

\section{Conclusions}

Extended maceration increased the concentration of phenolic compounds, color intensity, and antioxidant capacity in wine. Grape harvest influenced the phenolic composition and antioxidant capacity, with samples from the 2016 harvest showing the best results. This outcome could have been favored by climate conditions in those years, with higher synthesis and accumulation of phenolics in grapes, possibly contributing to high-quality wine. However, after 12 days of maceration, the phenolic content stabilized until the end of the maceration period. Little variation was noted for the color intensity. Thus, extended maceration between 16 and 20 days is recommended in order to enhance antioxidant activity due to the increase in the content of bioactive compounds.

\section{Acknowledgments}

The authors would like to acknowledge Empresa Brasileira de Pesquisa Agropecuária for the financial support, Fundação de Amparo a Pesquisa do Estado da Bahia (FAPESB) for granting a scholarship, and Santa Maria winery for providing the grapes.

\section{Conflict of interest}

The authors declare no conflict of interest.

\section{Supporting information}

Table S1. Monthly weather data of Campo experimental do bebedouro, Embrapa Semiárido, regarding two production cycles concerning the first and second semesters of 2016 and 2017, respectively, evaluated from pruning until harvest.

Table S2. Validation method parameters for identification and quantification of phenolic compounds performed by HPLC-DADFD.

Table S3. Equations for predictive models.

\section{References}

Alencar, N.M.M., Cazarin, C.B.B., Corrêa, L.C., Junior, A.R.M., Biasoto, A.C.T., and Behrens, J.H. (2018). Influence of maceration time on phenolic compounds and antioxidant activity of the Syrah must and wine. J. Food Biochem. 42: e12471.
Barbará, J.A., Silva, E.A.S., Biasoto, A.C.T., Gomes, A.A., Côrrea, L.C., Leão, P.C.S., and Zini, C.A. (2019). Maturation and Maceration Effects on Tropical Red Wines Assessed by Chromatography and Analysis of Variance - Principal Component Analysis. J. Braz. Chem. Soc. 30: 1357-1377.

Brand-Williams, W., Cuvelier, M.E., and Berset, C. (1995). Use of a free radical method to evaluate antioxidant activity. LWT - Food Sci. Technol. 28: 25-30.

Camargo, U.A., Pereira, G.E., and Guerra, C.C. (2011). Wine grape cultivars adaptation and selection for tropical regions. Acta Hortic. 910: 121-129.

Casassa, L.F., Beaver, C.W., Mireles, M.S., and Harbertson, J.F. (2013). Effect of extended maceration and ethanol concentration on the extraction and evolution of phenolics, color components and sensory attributes of Merlot wines. Aust. J. Grape and Wine Res. 19: 25-39.

Costa, R.R., Rodrigues, A.A.M., Vasconcelos, V.A.F., Costa, J.P.D., and Lima, M.A.C. (2020). Trellis systems, rootstocks and season influence on the phenolic composition of 'Chenin. Blanc' grape. Sci. Agric. 77: e20180207.

De Coninck, G., Jordão, A.M., Ricardo-Da-Silva, J.M., and Laureano, O. (2006). Evolution of phenolic composition and sensory properties in red wine aged in contact with Portuguese and French oak wood chips. J. Int. Sci. Vigne Vin. 40: 25-34.

Faitová, K., Hejtmánková, A., Lachman, J., Pivec, V., and Dudjak, J. (2004). The contents of total polyphenolic compounds and trans-resveratrol in white Riesling originated in the Czech Republic. Czech J. Food Sci. 22: 215-221.

Fernandez-Mar, M.I., Mateos, R., Garcia-Parrilla, M.C., Puertas, B., and Cantos-Villar, E. (2012). Bioactive compounds in wine: Resveratrol, hydroxytyrosol and melatonin: A review. Food Chem. 130: 797-813.

Garambone, E., and Rosa, G. (2008). Possíveis benefícios do ácido clorogênico à saúde. Alim. Nutr. 18: 229-235.

Garrido, J., and Borges, F. (2013). Wine and grape polyphenols chemical perspective. Food Res. Int. 54: 1844-1858.

Gil, M., Kontoudakis, N., González, E., Esteruelas, M., Fort, F., Canals, J.M., and Zamora, F. (2012). Influence of Grape Maturity and Maceration Length on Color, Polyphenolic Composition, and Polysaccharide Content of Cabernet Sauvignon and Tempranillo Wines. J. Agric. Food Chem. 60: 7988-8001.

Gómez-Cordovés, C., and González-Sanjosé, M.L. (1995). Interpretation of color variables during the aging of red wines: relationship with families of phenolic compounds. J. Agric. Food Chem. 43: 557-561.

Gomez-Miguez, M., Gonzalez-Miret, M.L., and Heredia, F.J. (2007). Evolution of colour and anthocyanin composition of Syrah wines elaborated with pre-fermentative cold maceration. J. Food Eng. 79: 271-278.

Gonçalves, F.J., and Jordão, A.M. (2009). Changes in antioxidant activity and proanthocyanidin fraction of red wine aged in contact with Portuguese (Quercus pyrenaica Willd.) and American (Quercus alba L.) oak wood chips. Ital. J. Food Sci. 21: 51-64.

González-Manzano, S., Rivas-Gonzalo, J.C., and Santos-Buelga, C. (2004). Extraction of flavan-3-ols from grape seed and skin into wine using simulated maceration. Anal. Chim. Act. 513: 283-289.

Granato, D., Carrapeiro, M.M., Fogliano, V., Saskia, M., and Ruth, V. (2016). Effects of geographical origin, varietal and farming system on the chemical composition and functional properties of purple grape juices: A review. Trends Food Sci. Technol. 52: 31-48.

Gris, E.F., Mattivi, F., Ferreira, E.A., Vrhovsek, U., Filho, D.W., Pedrosa, R.C., and Bordignon-Luiz, M.T. (2013). Phenolic profile and effect of regular consumption of Brazilian red wines on in vivo antioxidant activity. J. Food Compos. Anal. 31: 31-40.

Harbertson, J.F., and Spayd, S. (2006). Measuring phenolics in the Winery Am. J. Enol. Vitic. 3: 280-288.

Hermosin-Gutiérrez, I. (2003). Influence of ethanol content on the extent of copigmentation in a Cencibel young red wine. J. Agric. Food Chem. 51: 4079-4083.

Jackson, R.S. (2003). Modern biotechnology of winemaking. In: Sandler, M., and Pinder, R. (Ed.). Wine. Taylor and Francis, New York, NY, pp. 228-259.

Jordão, A.M., and Correia, A.C. (2012). Relationship Between Antioxidant Capacity, Proanthocyanidin and Anthocyanin Content During Grape Maturation of Touriga Nacional and Tinta Roriz Grape Varieties. 
South African J. Enol. 33: 214-223.

Jordão, A.M., Ricardo-Da-Silva, J.M., and Laureano, O. (2001). Evolution of catechin and procyanidin composition during grape maturation of two varieties (Vitis vinifera L.) Castelão Francês and Touriga Francesa. Am. J. Enol. Vit. 5: 230-234.

Kelebek, H., Canbas, A., Selli, S., Saucier, C., Jourdes, M., and Glories, Y. (2006). Influence of different maceration times on the anthocyanin composition of wines made from Vitis vinífera L. cvs. Boğazkere and Okuzgozu. J. Food Eng. 77: 1012-1017.

Kennedy, J.A. (2008). Grape and wine phenolics: Observations and recent findings. Cien. Investig. Agrar. 35: 107-120.

Kocabey, N.M., Yilmaztekin, M., and Hayaloglu, A.A. (2016). Effect of maceration duration on physicochemical characteristics, organic acid, phenolic compounds and antioxidant activity of red wine from Vitis vinifera L. Karaoglan. J. Food Sci Technol. 9: 3557-3565.

Kosseva, M.R., Joshi, V.K., and Panesar, P.S. (2017). Science and technology of fruit wine production. Academic Press Limited, San Diego, CA.

Leeuw, R., Kevers, C., Pincemail, J., Defraigne, J.O., and Dommes, J. (2014). Antioxidant capacity and phenolic composition of red wines from various grape varieties: Specificity of Pinot Noir. J. Food Compos. Anal. 36: 40-50.

Lima, M.S., Dutra, M.C.P., Toaldo, I.M., Corrêa, L.C., Pereira, G.E., Oliveira, D., and Ninow, J.L. (2015). Phenolic compounds, organic acids and antioxidant activity of grape juices produced in industrial scale by different processes of maceration. Food Chem. 188: 384-392.

Lingua, M.S., Fabani, M.P., Wunderlin, D.A., and Baroni, M.V. (2016). From grape to wine: Changes in phenolic composition and its influence on antioxidant activity. Food Chem. 208: 228-238.

Lucena, A.P.S., Nascimento, R.J.B., Maciel, J.A.C., Tavares, J.X., Barbosa-Filho, J.M., and Oliveira, E.J. (2010). Antioxidant activity and phenolics content of selected Brazilian wines. J. Food Compos. Anal. 23: 30-36.

Lukic, I., Budic-Leto, I., Bubola, M., Daijanic, K., and Staver, M. (2016). Pre-fermentative cold maceration, saignée, and various termal treatments as options for modulating volatile aroma and phenol profiles of red wine. Food Chem. 224: 51-261.

Mcrae, J.M., Teng, B., and Bindon, K. (2019). Factors Influencing Red Wine Color from the Grape to the Glass. In: Melton, L., Shahidi, F., and Varelis, P. (Ed.). Encyclopedia of Food Chemistry. Elsevier, pp. 97-106.

Monagas, M., Gomez-Cordoves, C., Bartolome, B., Laureano, O., and Silva, J.M.R. (2003). Monomeric, oligomeric, and polymeric flavan3-ol composition of wines and grapes from Vitis vinifera L. CV. Graciano, Tempranillo, and Cabernet Sauvignon. J. Agric. Food Chem. 51 6475-6481.

Moreno-Arribas, M.V., and Polo, C. (2008). Wine chemistry and biochemistry. Springer Verlag, New York.

Natividade, M.M.P., Corrêa, L.C., Souza, S.V.C., Pereira, G.E., and Lima, L.C.D. (2013). Microch. Jour. 110: 665-674.

Oliveira, J.B., Faria, D.L., Duarte, D.F., Egipto, R., Laureano, O., Castro, R., Pereira, G.E., and Silva, J.M.R. (2019). Efeito da época de colheita na composição fenólica e parâmetros enológicos de uvas e vinhos cv. 'Touriga Nacional' (Vitis vinifera L.) produzidos sob clima tropical Semiárido, no Estado de Pernambuco, Brasil. Ciênc. Téc. Vitiv. 33 145-166.

Oroian, M., and Escriche, I. (2015). Antioxidants: Characterization, natura sources, extraction and analysis. F. Res. Inter. 74: 10-36.

Padilha, C.V.S., Biasoto, A.C.T., Corrêa, L.C., Lima, M.S., and Pereira, G.E. (2017a). Phenolic compounds profile and antioxidant activity of commercial tropical red wines (Vitis vinifera L.) from São Francisco Valley, Brazil. J. Food Biochem. 41: 1-9.

Padilha, C.V.S., Miskinis, G.A., Souza, M.E.A.O., Pereira, G.E., Oliveira, D.M., Bordignon-Luiz, T., and Lima, M.S. (2017b). Rapid determination of flavonoids and phenolic acids in grape juices and wines by RP-HPLC/ DAD: Method validation and characterization of commercial products of the new Brazilian varieties of grape. Food Chem. 228: 106-115.

Pereira, G.E., Guerra, C.C., Amorim, F.M., Nascimento, A.M.S., Souza, J.F., Lima, L.L.A., Lima, M.S., Padilha, C.V.S., Protas, J.F.S., Zanus, M.C. and Tonietto, J. (2018). Vinhos tropicais do semiárido do Brasil: desvendando o potencial vitivinícola desta nova fronteira geográfica do vinho. Territoires du Vin. 9: 91-13.

Pereira, G.E., Padilha, C.V.S., Marques, A.T.B., Canuto, K.M., Mendes, A and Souza, J.F. (2016). Lepoidsdesconsommateurs sur l'evolution des vins: L'exemple de la Vallee Du Sao Francisco, Bresil. In: Perard, J., and Perrot, M. (Ed.). Vin et Civilisation lês étapes de l'humanisation. Centre Georges Chevrier, Dijon, France, pp. 301-310.

Peynaud, E. (1997). . Connaissance et Travail du Vin (2nd ed.). Dunod, Paris, pp. 134-145.

Ribéreau-Gayon, P., Dubourdie, D., Donèche, B., and Lonvaud, A. (2006) Handbook of Enology - The Microbiology of Wine and Vinifications (2nd ed.). Chichester, UK, John Wiley and Sons, Ltda.

Ribéreau-Gayon, Y.G., Maujean, A., and Dubourdieu, D. (2006). The chemistry of wine stabilization and treatments handbook of enology (2nd ed.). Wiley, London, pp. 96-104.

Roleira, F.M.F., Tavares-Da-Silva, E.J., Varela, C.L., Costa, S.C., Silva, T., Garrido, J., and Borges, F. (2015). Plant derived and dietary phenolic antioxidants: Anticancer properties. Food Chem. 183: 235-258.

Samoticha, J., Wojdylo, A., and Golis, T. (2017). Phenolic composition, physicochemical properties and antioxidant activity of interspecific hybrids of grapes growing in Poland. Food Chem. 215: 263-273.

Singleton, V.L., and Rossi, J.A. (1965). Colorimetry of total phenolics with phosphomolybdic phosphotungstic acid reagents. Am. J. Enol. Vit 16: $144-158$.

Sun, B.S., Ricardo-Da-Silva, J.M., and Spranger, I. (2001). Quantification of catechins and proanthocyanidin sinseveral Portuguese grape vine varieties and red wines. Cien. Téc Vitiviníc. 16: 23-34.

Teixeira, A.H.C., Scherer-Warren, M., Hernandez, F.B.T., Andrade, R.G., and Leivas, J.F. (2013). Large-scale water productivity assessments with MODIS images in a changing semiarid environment: A Brazilian case study. Remote Sens. 5: 5783-5804.

Tsanova-Savova, S., and Ribarova, F. (2012). Free and conjugated myricetin, quercetin, and kaempferol in Bulgarian red wines. J. Food Compos. Anal. 5: 639-645.

Vauzour, D., Rodriguez-Mateos, A., Corona, G., Oruna-Concha, M.J., and Spencer, J.P.E. (2010). Polyphenols and human health: Prevention of disease and mechanisms of action. Nutrients 2: 1106-1131.

Yilmaztekin, M., Kocabey, N., and Hayaloglu, A.A. (2015). Effect of maceration time on free and bound volatiles of red wines from cv. Karaoğlan (Vitis vinifera L.) grapes grown in Arapgir, Turkey. J. Food Sci. 80: C556-C563. 